\title{
On dual defective manifolds
}

\author{
Paltin Ionescu And Francesco Russo
}

\begin{abstract}
An embedded manifold is dual defective if its dual variety is not a hypersurface. Using the geometry of the variety of lines through a general point, we characterize scrolls among dual defective manifolds. This leads to an optimal bound for the dual defect, which improves results due to Ein. We also discuss our conjecture that every dual defective manifold with cyclic Picard group should be secant defective, of a very special type, namely a local quadratic entry locus variety.
\end{abstract}

\section{Introduction}

The present paper is a natural sequel to our previous work, see [Ru, IR, IR2, IR3] and also [BI]. Using the geometry of the variety of lines passing through the general point of an embedded projective manifold, we characterize scrolls among all dual defective manifolds, and relate the latter to some special secant defective ones.

We consider $n$-dimensional irreducible non-degenerate complex projective manifolds $X \subset \mathbb{P}^{n+c}$. We call $X$ a prime Fano manifold of index $i(X)$ if its Picard group is generated by the hyperplane section class $H$ and $-K_{X}=i(X) H$ for some positive integer $i(X)$. One consequence of Mori's work [Mo] is that, for $i(X) \geqslant \frac{n+1}{2}, X$ is covered by lines, i.e., through each point of $X$ there passes a line, contained in $X$. Denote by $a$ the dimension of the variety of lines (belonging to some irreducible covering family), that pass through a general point of $X$. The "biregular part" of Mori Theory (no singularities, no flips, etc.), see [Mo2, De, Ko], provides the natural setting for understanding such manifolds. For instance, as first noticed in [BSW], when $a \geqslant \frac{n-1}{2}$, there is a Mori contraction of the covering family of lines. Moreover, its general fiber (which is still covered by lines) has cyclic Picard group, thus being a prime Fano manifold. For prime Fanos, the study of covering families of lines is nothing but the classical aspect in the theory of the variety

2000 Mathematics Subject Classification. 14MXX, 14NXX, $14 \mathrm{~J} 45$.

Key words and phrases. Fano manifold, covered by lines, dual and secant defective, scroll. 
of minimal rational tangents, developed by Hwang and Mok in a remarkable series of papers; see e.g. [HM, HM2, HM3, Hw]. Recall that $X$ is dual defective if its dual variety is not a hypersurface. Prime Fanos of high index other than complete intersections are quite rare. For instance, dual defective manifolds and some special but important secant defective ones provide such examples. Thus, when $\operatorname{Pic}(X)=\mathbb{Z}\langle H\rangle$, the class of local quadratic entry locus varieties, see [Ru, IR, IR2], gives examples with $i(X)=\frac{n+\delta}{2}, \delta$ being the secant defect, while dual defective manifolds have $i(X)=\frac{n+k+2}{2}$, where $k$ is the dual defect, see [E2].

Starting from an observation due to $\mathrm{Buch}[\mathrm{Bu}]$, we show that when $a \geqslant n-c$, each line from the given irreducible covering family is part of the contact locus of a suitable hyperplane. We elaborate on this and prove that under some mild condition (which is, conjecturally, automatically fulfilled) for dual defective manifolds with cyclic Picard group each general line is a contact line. This allows us, using also [BFS], to characterize scrolls among all dual defective manifolds; see Theorem 3.4. As a consequence, we get an optimal bound on the dual defect, generalizing one of the main results in Ein's foundational papers [E2, E1]. We also give evidence for our conjecture asserting that dual defective manifolds with cyclic Picard group should be local quadratic entry locus varieties.

\section{Preliminaries}

\section{(*) Setting, terminology and notation}

Throughout the paper we consider $X \subset \mathbb{P}^{N}$ an irreducible complex projective manifold of dimension $n \geqslant 1 . X$ is assumed to be non-degenerate and $c$ denotes its codimension, so that $N=n+c . X \subset \mathbb{P}^{N}$ is called quadratic if it is scheme theoretically an intersection of quadrics. For $x \in X$ we let $\mathbf{T}_{x} X$ denote the (affine) Zariski tangent space to $X$ at $x$, and write $T_{x} X$ for its projective closure in $\mathbb{P}^{N}$. $H$ denotes a hyperplane section (class) of $X$. As usual, $K_{X}$ stands for the canonical class of $X$. Also, if $Y \subset$ $X$ is a submanifold, we denote by $N_{Y / X}$ its normal bundle. For a vector bundle $E, \mathbb{P}(E)$ stands for its projectivized bundle, using Grothendieck's convention.

We let $S X \subset \mathbb{P}^{N}$ be the secant variety of $X$, that is the closure of the locus of secant lines. The secant defect of $X$ is the (non-negative) number $\delta=\delta(X):=2 n+1-\operatorname{dim}(S X)$. We say $X$ is secant defective when $\delta>0$.

A secant defective manifold $X \subset \mathbb{P}^{N}$ is called a local quadratic entry locus manifold, LQELM for short, if any two general points $x, x^{\prime} \in X$ belong to 
a $\delta$-dimensional quadric $Q_{x, x^{\prime}} \subset X$. See $[\mathrm{Ru}, \mathrm{IR}]$ for a systematic study of these special secant defective manifolds.

$X \subset \mathbb{P}^{N}$ is conic-connected if any two general points $x, x^{\prime} \in X$ are contained in some conic $C_{x, x^{\prime}} \subset X$. Clearly, any LQELM is also conic-connected. Classification results for conic-connected manifolds are obtained in [IR2], working in the general setting of rationally connected manifolds.

$X \subset \mathbb{P}^{N}$ is dual defective, DD for short, if its dual variety $X^{*}$ is not a hypersurface in the dual projective space $\mathbb{P}^{N *}$. Its dual defect is the (positive) number $k:=N-1-\operatorname{dim}\left(X^{*}\right)$.

For a general point $x \in X$, we denote by $\mathcal{L}_{x}$ the (possibly empty) scheme of lines contained in $X$ and passing through $x$. We say that $X \subset \mathbb{P}^{N}$ is covered by lines if $\mathcal{L}_{x}$ is not empty for $x \in X$ a general point. We refer the reader to $[\mathrm{De}, \mathrm{Ko}]$ for standard useful facts about the deformation theory of rational curves; we shall use them implicitly in the simplest case, that is lines on $X$.

Recall that $X$ is Fano if $-K_{X}$ is ample. The index of $X$, denoted by $i(X)$, is the largest integer $j$ such that $-K_{X}=j A$ for some ample divisor $A$.

\section{(**) Some standard exact sequences}

Let $V$ be a complex vector space of dimension $N+1$ such that $\mathbb{P}(V)=\mathbb{P}^{N}$. Consider the restriction of the Euler sequence on $\mathbb{P}^{N}$ to $X$

$$
0 \rightarrow \Omega_{\mathbb{P}^{N} \mid X}^{1} \rightarrow V \otimes \mathcal{O}_{X}(-1) \rightarrow \mathcal{O}_{X} \rightarrow 0
$$

and the exact sequence on $X$

$$
0 \rightarrow N_{X / \mathbb{P}^{N}}^{*} \rightarrow \Omega_{\mathbb{P}^{N} \mid X}^{1} \rightarrow \Omega_{X}^{1} \rightarrow 0
$$

From these exact sequences we deduce

$$
0 \rightarrow N_{X / \mathbb{P}^{N}}^{*}(1) \rightarrow V \otimes \mathcal{O}_{X} \rightarrow \mathcal{P}_{X} \rightarrow 0
$$

and

$$
0 \rightarrow \Omega_{X}^{1}(1) \rightarrow \mathcal{P}_{X} \rightarrow \mathcal{O}_{X}(1) \rightarrow 0
$$

where $\mathcal{P}_{X}$ is the first jet bundle of $\mathcal{O}_{X}(1)$.

If $\mathbb{G}(n, N)$ denotes the Grassmannian of $n$-planes in $\mathbb{P}^{N}$, let $\gamma_{X}: X \rightarrow$ $\mathbb{G}(n, N)$ be the Gauss map of $X$, associating with a point $x \in X$ the point of $\mathbb{G}(n, N)$ corresponding to the projective tangent space $T_{x} X$ to $X$ at $x$. 
By Zak's theorem on Tangencies, the morphism $\gamma_{X}$ is finite and birational; see [Za, I.2.8].

Let $\mathcal{U}$ be the universal quotient bundle on $\mathbb{G}(n, N)$, which is a locally free sheaf of rank $n+1$. We have a natural surjection

$$
V \otimes \mathcal{O}_{\mathbb{G}(n, N)} \rightarrow \mathcal{U}
$$

inducing the surjection

$$
V \otimes \mathcal{O}_{X} \rightarrow \gamma_{X}^{*}(\mathcal{U})
$$

Then it is easy to see that $\mathcal{P}_{X}=\gamma_{X}^{*}(\mathcal{U})$; so, for every closed point $x \in X$ we have $\mathbb{P}\left(\mathcal{P}_{X} \otimes k(x)\right)=T_{x} X \subset \mathbb{P}(V)$.

The above surjection gives an embedding, over $X$, of $\mathbb{P}\left(\mathcal{P}_{X}\right) \rightarrow X$ into $\mathbb{P}(V) \times X \rightarrow X$ in such a way that the restriction $\pi_{1}$ of the projection $\mathbb{P}^{N} \times$ $X \rightarrow \mathbb{P}^{N}$ to $\mathbb{P}\left(\mathcal{P}_{X}\right)$ maps $\mathbb{P}\left(\mathcal{P}_{X}\right)$ onto $T X=\bigcup_{x \in X} T_{x} X$.

By (1.4) we get

$$
\gamma_{X}^{*}(\operatorname{det}(\mathcal{U}))=\operatorname{det}\left(\mathcal{P}_{X}\right) \simeq \omega_{X} \otimes \mathcal{O}_{X}(n+1) .
$$

The following simple lemma will provide useful.

Lemma 1.1. Let $E$ be a spanned vector bundle on $X$ and let $C \subset \mathbb{P}(E):=\mathbb{P}$ be a smooth curve, orthogonal to the tautological line bundle, $L$, of $\mathbb{P}$. Let $C^{\prime}$ be the projection of $C$ to $X$. The following relation between normal bundles holds: $\operatorname{deg}\left(N_{C^{\prime} / X}\right)=\operatorname{deg}\left(N_{C / \mathbb{P}}\right)+\operatorname{deg}\left(\left.E\right|_{C^{\prime}}\right)$.

Proof. Since $E$ is spanned, the linear system $|L|$ is base points free, defining a morphism $\varphi$. As $L$ restricted to any fiber of the projection $\pi: \mathbb{P} \rightarrow X$ is very ample, $\pi$ maps fibers of $\varphi$ isomorphically to $X$. In particular, $C$ projects isomorphically onto $C^{\prime}$, so $C^{\prime}$ is smooth. The result follows by combining the adjunction formula with the well-known expression for the canonical class of $\mathbb{P}$ :

$$
K_{\mathbb{P}}=-\operatorname{rk}(E) L+\pi^{*}\left(\operatorname{det}(E)+K_{X}\right) .
$$

\section{Manifolds covered by lines}

Let $X \subset \mathbb{P}^{N}$ be as in $(*)$. Fix some irreducible component, say $\mathcal{F}$, of the Hilbert scheme of lines on $X \subset \mathbb{P}^{N}$, such that $X$ is covered by the lines in $\mathcal{F}$. Put $a=: \operatorname{deg}\left(N_{\ell / X}\right)$, where $[\ell] \in \mathcal{F}$. For $x \in X$, let $\mathcal{F}_{x}=\{[\ell] \in \mathcal{F} \mid$ $x \in \ell\}$. Note that for $x \in X$ general and for $[\ell] \in \mathcal{F}_{x}$ we have $a \geqslant 0$ and 
$a=\operatorname{dim}_{[\ell]}\left(\mathcal{F}_{x}\right)$. We may view $\mathcal{F}_{x}$ as a closed subscheme of $\mathbb{P}\left(\left(\mathbf{T}_{x} X\right)^{*}\right) \cong$ $\mathbb{P}^{n-1}$.

When the dimension of $\mathcal{F}_{x}$ is large, the study of manifolds covered by lines is greatly simplified by the following two facts:

First, we may reduce, via a Mori contraction, to the case where the Picard group is cyclic; this is due to Beltrametti-Sommese-Wiśniewski, see [BSW]. Secondly, the variety $\mathcal{F}_{x} \subset \mathbb{P}^{n-1}$ inherits many of the good properties of $X \subset \mathbb{P}^{N}$; this is due to Hwang; see [Hw]. See [BI] for an application of these principles.

Theorem 2.1. Assume $a \geqslant \frac{n-1}{2}$. Then the following results hold:

(1) ([BSW]) There is a Mori contraction, say $\operatorname{cont}_{\mathcal{F}}: X \rightarrow W$, of the lines from $\mathcal{F}$; let $F$ denote a general fiber of $\operatorname{cont} \mathcal{F}$ and let $f$ be its dimension;

(2) ([Wi]) $\operatorname{Pic}(F)=\mathbb{Z}\left\langle H_{F}\right\rangle, i(F)=a+2$ and $F$ is covered by the lines from $\mathcal{F}$ contained in $F$;

(3) $([\mathrm{Hw}]) \mathcal{F}_{x} \subseteq \mathbb{P}^{f-1}$ is smooth irreducible non-degenerate. In particular, $F$ has only one maximal irreducible covering family of lines.

We refer now to the setting in $(* *)$. The following considerations are an elaboration of Lemma 1 from [Bu].

Consider a line $l \subset X$. Then $N_{l / X} \simeq \mathcal{O}_{\mathbb{P}^{1}}\left(a_{1}\right) \oplus \cdots \oplus \mathcal{O}_{\mathbb{P}^{1}}\left(a_{n-1}\right)$ with $a_{1} \leqslant a_{2} \leqslant \cdots \leqslant a_{n-1} \leqslant 1$. Let $a(l)=\#\left\{i \mid a_{i}=1\right\}$. If $l$ passes through a general point $x \in X$, then $a_{1} \geqslant 0$ and $\operatorname{deg}\left(N_{l / X}\right)=a(l)$.

For every line $l \subset X$ we shall consider the following linear spaces:

$$
\left\langle\bigcup_{x \in l} T_{x} X\right\rangle
$$

which is the linear span of the union of the tangent spaces at points $x \in l$ and

$$
\bigcap_{x \in l} T_{x} X
$$

By the discussion from $(* *)$, we also have the equality

$$
\pi_{1}\left(\mathbb{P}\left(\left(\mathcal{P}_{X}\right)_{\mid l}\right)\right)=\bigcup_{x \in l} T_{x} X
$$

Since we shall be interested in $\bigcup_{x \in l} T_{x} X$ and in the dimension of its linear span in $\mathbb{P}^{N}$, we shall analyze $\mathcal{P}_{X \mid l}$, where for the moment $l \subset X$ is 
an arbitrary line. From (1.3) we deduce that $\mathcal{P}_{X}$ and $\mathcal{P}_{X \mid l}$ are generated by global sections so that

$$
\mathcal{P}_{X \mid l} \simeq \bigoplus_{j=1}^{n+1-b_{0}(l)} \mathcal{O}_{\mathbb{P}^{1}}\left(b_{j}(l)\right) \bigoplus \mathcal{O}_{\mathbb{P}^{1}}^{b_{0}(l)}
$$

with $b_{j}(l)>0$ for every $j=1, \ldots, n+1-b_{0}(l)$.

Let $\Pi=\mathbb{P}^{b}=\mathbb{P}(U) \subset \mathbb{P}^{N}$. We have a surjection $V \rightarrow U$ inducing a surjection $V \otimes \mathcal{O}_{X} \rightarrow U \otimes \mathcal{O}_{X}$ and hence an inclusion, over $X, \mathbb{P}(U) \times X \subset$ $\mathbb{P}(V) \times X$. Given a subvariety $Y \subseteq X$ we have that $\Pi \subset T_{y} X$ for every $y \in Y$ if and only if the natural surjection $V \otimes \mathcal{O}_{Y} \rightarrow U \otimes \mathcal{O}_{Y}$ factorizes through $V \otimes \mathcal{O}_{Y} \rightarrow \mathcal{P}_{X \mid Y}$, that is if and only if there exists a surjection $\mathcal{P}_{X \mid Y} \rightarrow U \otimes \mathcal{O}_{Y}$. Thus for a line $l \subset X$ we obtain that if $\Pi=\mathbb{P}^{b} \subset T_{x} X$ for every $x \in l$, then $b \leqslant b_{0}(l)-1$.

Proposition 2.2 (cf. [Bu, Lemma1]). Let notation be as above and let $l \subset X$ be a line passing through a general point of $X$. Then:

(1)

$$
\mathcal{P}_{X \mid l} \simeq \mathcal{O}_{\mathbb{P}^{1}}^{a(l)+2} \oplus \mathcal{O}_{\mathbb{P}^{1}}(1)^{n-1-a(l)}
$$

and

$$
\operatorname{dim}\left(\left\langle\bigcup_{x \in l} T_{x} X\right\rangle\right)=N-h^{0}\left(N_{X / \mathbb{P}^{N}}^{*}(1)_{\mid l}\right)
$$

In particular, the variety $\bigcup_{x \in l} T_{x} X$ is isomorphic to a linear projection of a cone with vertex a linear space of dimension a $(l)+1$ over the Segre variety $\mathbb{P}^{1} \times \mathbb{P}^{n-2-a(l)}$ so that

$$
\operatorname{dim}\left(\left\langle\bigcup_{x \in l} T_{x} X\right\rangle\right) \leqslant \min \{N, 2 n-1-a(l)\}
$$

$(2)$

$$
\operatorname{dim}\left(\bigcap_{x \in l} T_{x} X\right)=a(l)+1
$$

and for $x, y \in l$ general we have

$$
T_{y} \widetilde{C}_{x}=\bigcap_{z \in l} T_{z} X
$$

where $\widetilde{C}_{x}$ is the irreducible component of the locus of lines through $x$ to which $l$ belongs. 
(3) $h^{0}\left(N_{X / \mathbb{P}^{N}}(-1)_{\mid l}\right)=N-a(l)-1$ and $h^{0}\left(N_{X / \mathbb{P}^{N}}^{*}(1)_{\mid l}\right) \geqslant a(l)+c+1-n$.

(4) The following conditions are equivalent:

(i) $h^{0}\left(N_{X / \mathbb{P}^{N}}^{*}(1)_{\mid l}\right)=a(l)+c+1-n$;

(ii) $\operatorname{dim}\left(\left\langle\bigcup_{x \in l} T_{x} X\right\rangle\right)=2 n-1-a(l)$;

(iii) the splitting-type of the bundle $N_{X / \mathbb{P}^{N}}(-1)_{\mid l}$ is $(0, \ldots, 0,1, \ldots, 1)$.

Proof. From the exact sequence

$$
0 \rightarrow \mathcal{O}_{l}(-1) \rightarrow \mathcal{P}_{X \mid l}^{*} \rightarrow T_{X}(-1)_{\mid l} \rightarrow 0
$$

and from $h^{0}\left(T_{X}(-1)_{\mid l}\right)=a(l)+2$ we deduce $b_{0}(l)=h^{0}\left(\mathcal{P}_{X \mid l}^{*}\right)=a(l)+2$. Moreover

$$
\begin{aligned}
n+1-b_{0}(l) \leqslant \sum_{j=1}^{n+1-b_{0}(l)} b_{j}(l) & =\operatorname{deg}\left(\mathcal{P}_{X \mid l}\right)=\left(K_{X}+(n+1) H\right) \cdot l \\
& =-a(l)-2+n+1=n+1-b_{0}(l)
\end{aligned}
$$

so that $b_{j}(l)=1$ for every $j=1, \ldots, n-1-a(l)$, proving the first assertion.

Let $\Pi=\bigcap_{x \in l} T_{x} X=\mathbb{P}^{b}$. By the previous analysis we deduce $b \leqslant a(l)+$ 1. If $l$ passes through the general point $x \in X$, consider $\widetilde{C}_{x}$, the irreducible component of the locus of lines through $x$ to which $l$ belongs, which is a cone whose vertex contains $x$. We know that $\operatorname{dim}\left(\widetilde{C}_{x}\right)=a(l)+1$ and that for every $y \in l$ obviously $T_{y} \widetilde{C}_{x} \subset T_{y} X$. Since $T_{y} \widetilde{C}_{x}$ does not depend on $y \in l$ for $y \in l$ general, we deduce that, for $x, y \in l$ general, $T_{y} \widetilde{C}_{x} \subseteq \bigcap_{z \in l} T_{z} X$. Therefore, $a(l)+1 \leqslant b$ which together with the previous inequality yields $b=a(l)+1$ and

$$
T_{y} \widetilde{C}_{x}=\bigcap_{z \in l} T_{z} X
$$

for $x, y \in l$ general points. This proves (2).

The assertions from (3) follow easily from the exact sequences in $(* *)$. The equivalence between (i) and (ii) from (4) is clear by the second assertion in (1). Write $\left.N_{X / \mathbb{P}^{N}}(-1)\right|_{l} \simeq \bigoplus_{j=1}^{c} \mathcal{O}_{\mathbb{P}^{1}}\left(d_{j}(l)\right)$, for non-negative integers $d_{j}(l)$. Let $d_{0}(l)=\#\left\{i \mid d_{i}=0\right\}$ and let $d_{1} \leqslant \cdots \leqslant d_{c}$.

We have the exact sequence

$$
\left.0 \rightarrow N_{l / X}(-1) \rightarrow N_{l / \mathbb{P}^{N}}(-1) \simeq \mathcal{O}_{l}^{N-1} \rightarrow N_{X / \mathbb{P}^{N}}(-1)\right|_{l} \rightarrow 0
$$


yielding

$$
\begin{aligned}
n-a(l)-1 & =-\operatorname{deg}\left(N_{l / X}(-1)\right)=\operatorname{deg}\left(\left.N_{X / \mathbb{P}^{N}}(-1)\right|_{l}\right) \\
& =\sum_{j=d_{0}(l)+1}^{c} d_{j} \geqslant c-d_{0}(l) .
\end{aligned}
$$

Moreover, equality holds in the last inequality if and only if condition (iii) from (4) is fulfilled. We deduce that $h^{0}\left(\left.N_{X / \mathbb{P}^{N}}^{*}(1)\right|_{l}\right)=d_{0}(l) \geqslant a(l)+c+$ $1-n$. This shows the equivalence between (i) and (iii).

Corollary 2.3. Keeping the notation and hypotheses from the previous proposition, we have:

(1) If the splitting-type of the bundle $N_{X / \mathbb{P}^{N}}(-1)_{\mid l}$ is $(0, \ldots, 0,1, \ldots, 1)$, then $a(l) \geqslant n-1-c$;

(2) If there is a line $l$ from a covering family such that $N_{X / \mathbb{P}^{N}}(-1)_{\mid l}$ is ample, then $a(l) \leqslant n-1-c$;

(3) If $X$ is quadratic, $n \geqslant c+2$ and there is a line l from a covering family such that $N_{X / \mathbb{P}^{N}}(-1)_{\mid l}$ is ample, then $X$ is a complete intersection.

Proof. (1) follows from the last point in the proposition. To see (2), recall that by the open nature of ampleness, we may assume that $l$ passes through the general point of $X$. So we may apply item (3) of the proposition, since $h^{0}\left(N_{X / \mathbb{P}^{N}}^{*}(1)_{\mid l}\right)=0$. The last point follows from [IR3], Theorem $3.8(3)$, using also the next remark.

Remark 2.4. If $X$ is quadratic and covered by lines, $N_{X / \mathbb{P}^{N}}^{*}(2)$ is spanned, so the splitting-type of the bundle $N_{X / \mathbb{P}^{N}}(-1)_{\mid l}$ is $(0, \ldots, 0,1, \ldots, 1)$, for any line $l$.

\section{Dual defective manifolds}

For $X \subset \mathbb{P}^{N}$, let $X^{*} \subset \mathbb{P}^{N *}$ be the dual variety of $X$ and let $\operatorname{def}(X)=N-$ $1-\operatorname{dim}\left(X^{*}\right)$ be the dual defect of $X$.

Working in different settings, Mumford in [Mum] and Landman (unpublished) called the attention on this very special but intriguing class of embedded manifolds. They have since then been studied thoroughly by Ein in [E2, E1] and by Beltrametti-Fania-Sommese in [BFS]. See also [LS, Mu, Mu2]. 
For a hyperplane $H \subset \mathbb{P}^{N}$ we define the contact locus of $H$ on $X$ as

$$
L=L(H)=\left\{x \in X \mid T_{x} X \subseteq H\right\}=\operatorname{Sing}(X \cap H) \subset X .
$$

If $[H] \in X^{*}$ corresponds to a smooth point in $X^{*}$, then by reflexivity $L(H) \simeq$ $\mathbb{P}^{\operatorname{def}(X)}$ is an embedded linear subspace of $\mathbb{P}^{N}$ contained in $X$. Any line $l \subset L(H)$ with $[H] \in X^{*}$ is called a contact line on $X$. We recall a basic result on the geometry of contact lines proved differently by Ein in [E2, Section 2].

Proposition 3.1 ([E2]). Let $k=\operatorname{def}(X)>0$ and let $l \subset L(H)$ be a contact line with $[H] \in X^{*}$ general. Then $N_{l / X} \simeq \mathcal{O}_{\mathbb{P}^{1}}^{\frac{n-k}{2}} \oplus \mathcal{O}_{\mathbb{P}^{1}}(1)^{\frac{n+k-2}{2}} ;$ in particular, $n$ and $k$ have the same parity, as first proved by Landman.

Proof. Apply Lemma 1.1 to a line $l \subset L(H)$, where $[H] \in X^{*}$ is general. Recall that the dual variety is obtained as the image of the natural map (given by the tautological line bundle) from the conormal variety $\mathbb{P}\left(N_{X / \mathbb{P}}\right.$ $(-1))$ to $\mathbb{P}^{N *}$. Seeing $l$ as contained in the linear $L(H) \simeq \mathbb{P}^{k}$ which is the fiber of that map, the degree of its normal bundle is $k-1$. Also, $\operatorname{deg}\left(N_{l / X}\right)=$ $a$ and $\operatorname{deg}\left(\left.N_{X / \mathbb{P}}(-1)\right|_{l}\right)=-\operatorname{deg}\left(N_{l / X}(-1)\right)=n-a-1$, see 2.6. The result follows.

Let $k=\operatorname{def}(X)>0$ and let $x \in X$ be a general point. By Proposition 3.1 there exists a unique irreducible component of $\mathcal{L}_{x}$ of dimension $\frac{n+k-2}{2}$ containing a given general contact line. The union of all these irreducible components of $\mathcal{L}_{x}$ is called $C_{x}$. Since $\mathcal{L}_{x}$ is smooth and since $\frac{n+k-2}{2} \geqslant \frac{n-1}{2}$, there exists a unique irreducible component of $\mathcal{L}_{x}$ containing all the general contact lines passing through $x$, i.e., $C_{x} \subseteq \mathcal{L}_{x}$ is an irreducible component of $\mathcal{L}_{x}$ of dimension $\frac{n+k-2}{2}$ and hence an irreducible smooth subvariety of $\mathcal{L}_{x}$. Easy examples like the Segre varieties $\mathbb{P}^{1} \times \mathbb{P}^{n-1} \subset \mathbb{P}^{2 n-1}$ show that in general $C_{x} \subsetneq \mathcal{L}_{x}$. For every line $[l] \in C_{x}$, we get $a(l)=\frac{n+k-2}{2}$.

Proposition 3.2. Let $X \subset \mathbb{P}^{N}$ be as in $(*)$ with $\operatorname{def}(X)=k>0$ and let $x \in X$ be a general point. If $k \geqslant n-2 c+2$, then for a general line $[l] \in C_{x}$

$$
\operatorname{dim}\left(\left\langle\bigcup_{x \in l} T_{x} X\right\rangle\right)=\frac{3 n-k}{2} \quad \text { and } \quad N_{X / \mathbb{P}^{N}}(-1)_{\mid l} \simeq \mathcal{O}_{\mathbb{P}^{1}}^{\frac{k+2 c-n}{2}} \oplus \mathcal{O}_{\mathbb{P}^{1}}(1)^{\frac{n-k}{2}}
$$

Moreover, every line $[l] \in C_{x}$ is a contact line and for a general $[l] \in C_{x}$ every hyperplane $H$ such that $l \subset L(H)$ represents a smooth point of $X^{*}$. In particular, through a general line $[l] \in C_{x} \subset \mathbb{P}^{n-1}$ there passes a linearly embedded $\mathbb{P}^{k-1}$ contained in $C_{x}$, corresponding to lines in $L(H)$ passing through $x$. 
Proof. If $[l] \in C_{x}$, then $a(l)=\frac{n+k-2}{2}$ so that the hypothesis and Proposition 2.2 imply

$$
\operatorname{dim}\left(\left\langle\bigcup_{x \in l} T_{x} X\right\rangle\right) \leqslant 2 n-1-a(l) \leqslant N-1
$$

Thus every line $[l] \in C_{x}$ is contained in the contact locus of at least one hyperplane $H$ with $[H] \in\left(T_{x} X\right)^{*}$.

Let $X_{x}^{*}=\left\{[H] \in X^{*} \mid T_{x} X \subseteq H\right\}=\left(T_{x} X\right)^{*} \simeq \mathbb{P}^{c-1}$. Put

$$
Z_{x}=\{([l],[H]) \mid l \subset L(H)\} \subset C_{x} \times X_{x}^{*}
$$

where $p_{x}: Z_{x} \rightarrow C_{x}$ is the restriction of the first projection and $q_{x}: Z_{x} \rightarrow X_{x}^{*}$ the restriction of the second projection. For every $[H] \in \operatorname{Sm}\left(X^{*}\right) \cap X_{x}^{*}$ we have $q_{x}^{-1}([H])=\mathbb{P}^{k-1}$, where $\operatorname{Sm}\left(X^{*}\right)$ denotes the smooth locus of $X^{*}$. Thus $q_{x}$ is surjective and every irreducible component of $Z_{x}$ dominating $X_{x}^{*}$ has dimension $k+c-2$. By (3.2) also $p_{x}$ is surjective and by definition of $Z_{x}$ and $C_{x}$ every irreducible component of $Z_{x}$ dominating $C_{x}$ dominates $X_{x}^{*}$ and vice versa. Let $U=q_{x}^{-1}\left(\operatorname{Sm}\left(X^{*}\right) \cap X_{x}^{*}\right)$. Since $p_{x}$ is proper and surjective, there exists an open subset $V \subseteq C_{x}$ such that $p_{x}^{-1}(V) \subseteq U$. Thus for every $[l] \in V$, every hyperplane $H$ such that $l \subset L(H)$ is a smooth point of $X^{*}$ and through $[l]$ there passes a linear embedded $\mathbb{P}^{k-1} \subset \mathbb{P}^{n-1}$ contained in $C_{x}$.

Let $[l] \in C_{x}$ be an arbitrary line and let $F_{[l]}=p_{x}^{-1}([l])$. By definition $F_{[l]}=\left(\left\langle\bigcup_{x \in l} T_{x} X\right\rangle\right)^{*}=\mathbb{P}^{N-b(l)-1}$, where $b(l)=\operatorname{dim}\left(\left\langle\bigcup_{x \in l} T_{x} X\right\rangle\right)$. Thus for a general $[l] \in C_{x}$

$$
\operatorname{dim}\left(F_{[l]}\right)=k+c-2-\frac{n+k-2}{2}=\frac{k+2 c-2-n}{2},
$$

and

$$
n+c-b(l)-1=N-b(l)-1=\operatorname{dim}\left(F_{[l]}\right)=\frac{k+2 c-2-n}{2},
$$

yielding $b(l)=\operatorname{dim}\left(\left\langle\bigcup_{x \in l} T_{x} X\right\rangle\right)=\frac{3 n-k}{2}=2 n-1-a(l)$.

The decomposition $N_{X / \mathbb{P}^{N}}(-1)_{\mid l} \simeq \mathcal{O}_{\mathbb{P}^{1}}^{\frac{k+2 c-n}{2}} \oplus \mathcal{O}_{\mathbb{P}^{1}}(1)^{\frac{n-k}{2}}$ follows now from Proposition 2.2 (4) and the rest is clear.

Lemma 3.3. Let $X \subset \mathbb{P}^{N}$ be as in (*) with $\operatorname{def}(X)=k>0$. If $k \geqslant \frac{n}{3}$, then $k \geqslant n-2 c+2$. 
Proof. By Zak's Theorem on Tangencies, $k \leqslant c-1$. Then

$$
n \leqslant 3 k \leqslant k+2 c-2
$$

concluding the proof.

Let us recall that $X \subset \mathbb{P}^{N}$ is an $r$-scroll, if $X \simeq \mathbb{P}(E)$ where $E$ is a rank $r+1$ vector bundle over some manifold $W$ and the fibers of the projection $\pi: \mathbb{P}(E) \rightarrow W$ are linearly embedded in $\mathbb{P}^{N}$. When $r>\operatorname{dim}(W)$, the scroll $X$ is dual defective and its defect equals $r-\operatorname{dim}(W)$. The next result characterizes scrolls among all dual defective manifolds.

Theorem 3.4. Let $X \subset \mathbb{P}^{N}$ be as in $(*)$ with $\operatorname{def}(X)=k>0$. Let $C_{x} \subset$ $\mathbb{P}^{n-1}$ be as above and put $\mathbb{P}^{m} \simeq T \subseteq \mathbb{P}^{n-1}$ to be the linear span of $C_{x}$ in $\mathbb{P}^{n-1}$. The following conditions are equivalent:

(i) $X$ is an $\frac{n+k}{2}$-scroll over a manifold of dimension $\frac{n-k}{2}$;

(ii) $\operatorname{dim}\left(C_{x}\right)>2 \operatorname{codim}_{T}\left(C_{x}\right)$ (or equivalently $k>\frac{4 m+6-3 n}{3}$ ).

Proof. We only have to prove that (ii) implies (i). Since $n \equiv k$ modulo 2 , it follows that $\operatorname{dim}\left(C_{x}\right)=\frac{n+k-2}{2} \geqslant\left[\frac{n}{2}\right]$. In particular, the lines in $C_{x}$ generate an extremal ray of $X$ by Theorem $2.1(1)$. Let $\varphi: X \rightarrow W$ be the contraction of this ray and let $F$ be a general fiber of $\varphi$. Then $F \subset \mathbb{P}^{N}$ is a smooth irreducible projective variety $\operatorname{such}$ that $\operatorname{Pic}(F) \simeq \mathbb{Z}\left\langle H_{F}\right\rangle$. Let $f=\operatorname{dim}(F)$ and let $\langle F\rangle$ be the linear span of $F$ in $\mathbb{P}^{N}$. Then $C_{x} \subseteq \mathbb{P}\left(\left(\mathbf{T}_{x} F\right)^{*}\right)=\mathbb{P}^{f-1}$ is smooth irreducible non-degenerate by Theorem 2.1 (3). Thus $m=f-1$. Moreover, by [BFS, Theorem (1.2)] we have $\operatorname{def}(F)=k(F)=k+n-f$, so that the hypothesis in (ii) yields $k(F)>\frac{f+2}{3}$. By Lemma 3.3 and Proposition 3.2 every line in $C_{x}$ is a contact line for $F \subseteq\langle F\rangle$ and $C_{x} \subseteq \mathbb{P}^{f-1}$ is covered by linear spaces of dimension $k(F)-1>\left[\frac{\operatorname{dim}\left(C_{x}\right)}{2}\right]$. From [Sa] (see also [BI] for a simple proof in the spirit of the present paper) it follows that $C_{x} \subseteq$ $\mathbb{P}\left(\left(\mathbf{T}_{x} F\right)^{*}\right)=\mathbb{P}^{f-1}$ is a scroll. The condition in (ii) and the Barth-Larsen Theorem, see $[\mathrm{BL}]$, give $\operatorname{Pic}\left(C_{x}\right) \simeq \mathbb{Z}\left\langle H_{C_{x}}\right\rangle$. So we get $C_{x}=\mathbb{P}^{f-1}$, hence $F=\mathbb{P}^{f}$ and $k(F)=f$. Therefore $f=\frac{n+k}{2}>\frac{n}{2}$ and $X \subset \mathbb{P}^{N}$ is a scroll by [E1, Theorem 1.7].

Corollary 3.5. Let $X \subset \mathbb{P}^{N}$ be as in $(*)$, with $\operatorname{def}(X)=k>0$. Let $\varphi$ : $X \rightarrow W$ be the contraction whose existence is ensured by Theorem 2.1 (1). 
Assume that $X$ is not a scroll. Then

$$
k \leqslant \frac{n+2-4 \operatorname{dim}(W)}{3} \leqslant \frac{n+2}{3} .
$$

Moreover:

(1) $k=\frac{n+2}{3}$ if and only if $N=15, n=10$ and $X \subset \mathbb{P}^{15}$ is projectively equivalent to the ten-dimensional spinorial variety $S^{10} \subset \mathbb{P}^{15}$.

(2) If $\operatorname{dim}(W)>0$, then $k=\frac{n+2-4 \operatorname{dim}(W)}{3}$ if and only if $\operatorname{dim}(W)=n-$ $10 \leqslant 3$ and $\varphi: X \rightarrow W$ is a fibration such that the general fiber $F \subset$ $\langle F\rangle \subset \mathbb{P}^{N}$ is isomorphic to $S^{10} \subset \mathbb{P}^{15} \subset \mathbb{P}^{N}$.

Proof. Keeping the notation from the preceding theorem, we have $k \leqslant$ $\frac{4 m+6-3 n}{3}, m=f-1$ and $f=n-\operatorname{dim}(W)$. So we may assume that $k=\frac{n+2}{3}$. Then $\operatorname{dim}\left(C_{x}\right)=\frac{2(n-1)}{3}$. By Lemma 3.3 and Proposition $3.2 C_{x} \subset \mathbb{P}^{n-1}$ is covered by linear spaces of dimension $k-1=\frac{n-1}{3}=\frac{\operatorname{dim}\left(C_{x}\right)}{2}$. Then by [Sa, NO] the variety $C_{x} \subset \mathbb{P}^{n-1}$ is one of the following:

(a) a scroll;

(b) a quadric hypersurface of even dimension;

(c) $\mathbb{G}(1, r)$ Plücker embedded with $r \geqslant 4$, or one of its isomorphic projections.

If $n<7$, then $n=4$ and $k=2=\operatorname{dim}\left(C_{x}\right)$; so, $i(X)=4=n$ and $X$ is a hyperquadric by the Kobayashi-Ochiai Theorem. This contradicts the fact that $X$ is DD. If $C_{x} \subset \mathbb{P}^{n-1}$ is a (quadric) hypersurface, then $\frac{2(n-1)}{3}=n-2$ yields $n=4$, obtaining once again a contradiction. Thus we can suppose $n \geqslant 7$, so that $\operatorname{Pic}\left(C_{x}\right)=\mathbb{Z}\langle H\rangle$ by the Barth-Larsen theorem. Therefore we are necessarily in case c). Since the secant defect of $\mathbb{G}(1, r)$ is four, we get: $n-1 \geqslant 2 \operatorname{dim}\left(C_{x}\right)+1-\delta\left(C_{x}\right)=\frac{4 n-13}{3}$, yielding $n \leqslant 10$. On the other hand, we have $\operatorname{dim}\left(C_{x}\right)=2(r-1) \geqslant 6$, so that $n \geqslant 10$. Therefore, $n=10$, $k=4$ and $i(X)=8$; moreover, since we assumed $k=\frac{n+2}{3}$, it follows that $\operatorname{dim}(W)=0$, so $F=X$, i.e., $\operatorname{Pic}(X) \simeq \mathbb{Z}\langle H\rangle$. The conclusion of part (1) follows by Mukai's classification of prime Fano manifolds of index $n-2$, see $[\mathrm{Muk}]$.

To prove (2), assume that $k=\frac{n+2-4 \operatorname{dim}(W)}{3}$. By [BFS, Theorem (1.2)]

$$
k(F)=\frac{n+2-4 \operatorname{dim}(W)}{3}+\operatorname{dim}(W)=\frac{f+2}{3},
$$


so that by the first part $f=10=n-\operatorname{dim}(W)$ and $F \subset\langle F\rangle \subset \mathbb{P}^{N}$ is isomorphic to $S^{10} \subset \mathbb{P}^{15}$.

Remark 3.6. The preceding corollary improves one of the main results in [E1] stating that if $k>0$ and $X$ is not a scroll, we have $k \leqslant \frac{n-2}{2}$, with equality only if $X$ is projectively isomorphic either to $\mathbb{G}(1,4) \subset \mathbb{P}^{9}$ or to $S^{10} \subset \mathbb{P}^{15}$. If $n \leqslant 9$ Ein's bound is better than ours; these cases may be recovered by our method, too.

\section{DD versus LQEL manifolds}

We begin by recalling the main known results about LQEL manifolds; complete proofs of the statements in the next theorem may be found in [Ru], Theorem 2.3, Corollaries 3.1, 3.2, [IR], Theorem 2.1, Propositions 3.2, 3.4 and [IR2], Theorem 2.2, Corollary 2.3.

Theorem 4.1. Assume $X \subset \mathbb{P}^{N}$ is a LQEL manifold of secant defect $\delta$. Then:

(1) $X$ is a Fano rational manifold with $\operatorname{rkPic}(X) \leqslant 2$.

(2) If $\operatorname{rkPic}(X)=2$ then $X$ is one of:

(a) $\mathbb{P}^{a} \times \mathbb{P}^{b}$ in its Segre embedding, or

(b) the hyperplane section of the above, or

(c) the blowing-up of $\mathbb{P}^{n}$ with center a linear space L, embedded by the linear system of quadrics through $L$.

(3) If $\operatorname{rkPic}(X)=1, X \cong v_{2}\left(\mathbb{P}^{n}\right)$ or $\operatorname{Pic}(X)=\mathbb{Z}\langle H\rangle$ and $i(X)=\frac{n+\delta}{2}$.

(4) If $\delta \geqslant 3$, then $\mathcal{L}_{x} \subset \mathbb{P}^{n-1}$ is again a LQELM, SL $\mathcal{L}_{x}=\mathbb{P}^{n-1}, \operatorname{dim}\left(\mathcal{L}_{x}\right)=$ $\frac{n+\delta}{2}-2, \delta\left(\mathcal{L}_{x}\right)=\delta-2$. If $\delta \geqslant \frac{n}{2}$, a complete classification is obtained.

(5) $X$ is a complete intersection if and only if $X$ is a quadric $\mathbb{Q}^{n}(\delta=n)$.

Proposition 4.2. Assume $X \subset \mathbb{P}^{N}$ is a LQEL manifold of secant defect $\delta$, different from $\mathbb{Q}^{n}$. Then $\delta \leqslant c+1$.

Proof. Observe first that the conclusion is clear if $\delta \leqslant 2$. So, we may assume $\delta \geqslant 3$. Let $a:=\operatorname{dim}\left(\mathcal{L}_{x}\right)$ and recall from Theorem 4.1 (4) that $a=\frac{n+\delta-4}{2}$. We claim that $a \geqslant n-c$. Indeed, if not, we would have $n-c+1 \leqslant \delta \leqslant n-$ $2 c+2$, so $c=1$, a contradiction. Thus, we may apply [MMT], Proposition 3.1 , giving $a \leqslant \frac{n+c-3}{2}$. As $\delta=2 a+4-n$, the conclusion follows. 
Corollary 4.3 (cf. [Ru, Remark 3.3]). The Hartshorne Conjecture on complete intersections, see [Ha], holds for LQEL manifolds.

Proof. We have $n-c+1 \leqslant \delta \leqslant c+1$, unless $X$ is a quadric. It follows that $n \leqslant 2 c$.

We also get a new proof of a bound on $\delta$ due to $\mathrm{B}$. $\mathrm{Fu}[\mathrm{Fu}]$. It expresses the fact that the Hartshorne Conjecture holds for the variety of lines of a LQEL manifold (see [IR3]).

Corollary 4.4 ([Fu]). Assume $X \subset \mathbb{P}^{N}$ is a LQEL manifold of secant defect $\delta$, different from $\mathbb{Q}^{n}$. Then $\delta \leqslant \frac{n+8}{3}$.

Proof. We may assume $\delta \geqslant 3$. From Theorem 4.1 (4), it follows that $\mathcal{L}_{x} \subset$ $\mathbb{P}^{n-1}$ is also a LQEL manifold. Moreover, it is easy to see that $\mathcal{L}_{x}$ is a quadric only if $X$ is. By the preceding Corollary applied to $\mathcal{L}_{x} \subset \mathbb{P}^{n-1}$, we get $a \leqslant 2(n-1-a)$, where $\operatorname{dim} \mathcal{L}_{x}=a=\frac{n+\delta-4}{2}$. The bound on $\delta$ follows.

Conjecture 4.5 ([IR2]). Assume that $X$ is a LQELM with $\operatorname{Pic}(X) \cong$ $\mathbb{Z}\langle H\rangle$. Then $X$ is obtained by linear sections and/or isomorphic projections from a rational homogeneous manifold, in its natural minimal embedding.

We recall that (linearly normal) rational homogeneous manifolds are well understood. In particular, those which are secant defective are known to be LQEL manifolds and are completely classified, see [Ka, Za]. They turn out to be quadratic manifolds and moreover we have that $\delta \leqslant 8$ if $X$ is not a quadric.

Conjecture 4.6 ([IR2]). Any dual defective manifold with cyclic Picard group is a LQELM.

Theorem (1.2) from [BFS], based on Theorem $2.1(1)$, reduces the study of dual defective manifolds to the case when $\operatorname{Pic}(X) \simeq \mathbb{Z}\langle H\rangle$. Note that $k \leqslant 4$ in all known examples other than scrolls.

The Conjecture 4.6 may be cut into two parts, stated as follows:

(1) any dual defective manifold with cyclic Picard group is conicconnected, and

(2) a dual defective manifold $X \subset \mathbb{P}^{N}$ as in (*) with cyclic Picard group and dual defect $k$, satisfies:

$$
k \geqslant n-c-1 \text {. }
$$


Note that if $X$ is both DD and LQELM, with cyclic Picard group, the formula for $\operatorname{dim}\left(\mathcal{L}_{x}\right)$ gives $\delta=k+2$. So, the condition in (2) corresponds to the (obvious) inequality $\delta \geqslant n-c+1$. Moreover, from [IR, Proposition 3.2] it follows that (1) and (2) together imply that $X$ is a LQELM.

Remarks 4.7. (1) Condition (2) above implies the inequality $k \geqslant n-$ $2 c+2$, which is the hypothesis in Proposition 3.2 (use that $n \equiv k$ modulo 2).

(2) By Zak's Theorem on Tangencies, we have $k \leqslant c-1$, which combined with the inequality in (2) yields $n \leqslant 2 c$ for dual defective manifolds. This would prove that DD manifolds satisfy the Hartshorne Conjecture. Note that all known linearly normal DD manifolds (with cyclic Picard group) are quadratic, so satisfy the Hartshorne Conjecture, as proved in [IR3].

(3) Knowing that (2) holds would lead to a much simpler proof of Corollary 3.5, without making use of the elaborate results from [Sa, NO].

(4) If $X$ has cyclic Picard group and is both dual defective and a LQELM, we have seen that $\delta=k+2$; therefore, the upper bounds on $\delta$ in Corollary 4.4, and on $k$ in Corollary 3.5, are the same. They express the condition that

$$
\operatorname{dim}\left(\mathcal{L}_{x}\right) \leqslant 2 \operatorname{codim}\left(\mathcal{L}_{x}, \mathbb{P}^{n-1}\right)
$$

that is the Hartshorne condition for the variety of lines.

\section{Acknowledgments}

The first author was partially supported by the Italian Program "Incentivazione alla mobilità di studiosi stranieri e italiani residenti all'estero" and by the grant PN-II-ID-PCE-2011-3-0288 (contract no. 132/05.10.2011). Both authors were partially supported by the Progetto PRIN Geometria sulle varietà algebriche.

\section{References}

[BL] W. Barth and M.E. Larsen, On the homotopy groups of complex projective algebraic manifolds, Math. Scand. 30 (1972), 88-94. 
[BFS] M.C. Beltrametti, M.L. Fania and A.J. Sommese, On the discriminant variety of a projective manifold, Forum Math. 4 (1992), $529-547$.

[BI] M.C. Beltrametti and P. Ionescu, On manifolds swept out by high dimensional quadrics, Math. Z. 260 (2008), 229-234.

[BSW] M.C. Beltrametti, A.J. Sommese and J. Wiśniewski, Results on varieties with many lines and their applications to adjunction theory, in 'Complex Algebraic Varieties' (Bayreuth, 1990), Lecture Notes in Mathematics, vol. 1507, Springer, 1992, pp. 16-38.

[Bu] A. Buch, Quantum cohomology of Grassmannians, Compos. Math. 137 (2003), 227-235.

[De] O. Debarre, Higher-dimensional algebraic geometry, Universitext, Springer, 2001.

[E1] L. Ein, Varieties with small dual variety. II, Duke Math. J. 52 (1985), 895-907.

[E2] — Varieties with small dual variety. I, Invent. Math. 86 (1986), $63-74$.

[Fu] B. Fu, Inductive characterizations of hyperquadrics, Math. Ann. 340 (2008), 185-194.

[Ha] R. Hartshorne, Varieties of small codimension in projective space, Bull. Amer. Math. Soc. 80 (1974), 1017-1032.

[Hw] J.M. Hwang, Geometry of minimal rational curves on Fano manifolds, in 'School on Vanishing Theorems and Effective Results in Algebraic Geometry (Trieste, 2000)', ICTP Lecture Notes, vol. 6, Abdus Salam Int. Cent. Theoret. Phys., 2001, pp. 335-393.

[HM] J.M. Hwang and N. Mok, Rigidity of irreducible Hermitian symmetric spaces of the compact type under Kähler deformation, Invent. Math. 131 (1998), 393-418.

[HM2] - Birationality of the tangent map for minimal rational curves, Asian J. Math. 8 (2004), 51-63.

[HM3] - Prolongations of infinitesimal linear automorphisms of projective varieties and rigidity of rational homogeneous spaces of Picard number 1 under Kähler deformation, Invent. Math. 160 (2005), 591-645. 
[IR] P. Ionescu and F. Russo, Varieties with quadratic entry locus. II, Compos. Math. 144 (2008), 949-962.

[IR2] —, Conic-connected manifolds, J. Reine Angew. Math. 644 (2010), 145-157.

[IR3] - Manifolds covered by lines and the Hartshorne Conjecture for quadratic manifolds, Amer. J. Math. 135 (2013), 349-360.

[Ka] H. Kaji, Homogeneous projective varieties with degenerate secants, Trans. Amer. Math. Soc. 351 (1999), 533-545.

[Ko] J. Kollár, Rational curves on algebraic varieties, Ergeb. Math. Grenzgeb. (3), vol. 32, Springer, 1996.

[LS] A. Lanteri and D. Struppa, Projective 7-folds with positive defect, Compos. Math. 61 (1987), 329-337.

[MMT] S. Marchesi, A. Massarenti and S. Tafazolian, Covered by lines and conic connected varieties, Le Matematiche LXVI (2011), 137-151.

[Mo] S. Mori, Projective manifolds with ample tangent bundle, Ann. Math. 110 (1979), 593-606.

[Mo2] - Threefolds whose canonical bundles are not numerically effective, Ann. Math. 116 (1982), 133-176.

[Muk] S. Mukai, Biregular classification of Fano 3-folds and Fano manifolds of coindex 3, Proc. Natl. Acad. Sci. USA 86 (1989), 3000-3002.

[Mum] D. Mumford, Some footnotes to the work of C. P. Ramanujam, in 'C. P. Ramanujam — a tribute', Springer, 1978, pp. 247-262.

[Mu] R. Muñoz, Varieties with low dimensional dual variety, Manuscripta Math. 94 (1997), 427-435.

[Mu2] — Varieties with degenerate dual variety, Forum Math. 13 (2001), 757-779.

[NO] C. Novelli and G. Occhetta, Projective manifolds containing a large linear space with nef normal bundle, Michigan Math. J. 60 (2011), 441-462.

[Ru] F. Russo, Varieties with quadratic entry locus. I, Math. Ann. 344 (2009), 597-617.

[Sa] E. Sato, Projective manifolds swept out by large-dimensional linear spaces, Tohoku Math. J. 49 (1997), 299-321. 
[Wi] J. Wiśniewski, On a conjecture of Mukai, Manuscripta Math. 68 (1990), 135-141.

[Za] F.L. Zak, Tangents and Secants of algebraic varieties, Transl. Math. Monogr., vol. 127, Amer. Math. Soc., 1993.

DipARTimento Di MATEMATICA

Università Degli Studi di Ferrara, VIa Machiavelli, 35, 44121 Ferrara

ITALY

and

Institute of Mathematics of the Romanian Academy

P.O. Box 1-764, 014700 BuCHAREST

ROMANIA

E-mail address: Paltin. Ionescu@unife.it

Dipartimento di Matematica e InFormatica

Università DEGLi Studi di CATANiA

Viale A. Doria, 6

95125 CATANIA

ITALY

E-mail address: frusso@dmi.unict.it

Received September 7, 2012 